\title{
(2) OPEN ACCESS \\ Evolving treatment landscape: 18 years of managing melanoma in a single patient
}

\author{
Garance Biosse-Duplan (10, ${ }^{1}$ Suzanne Murphy, ${ }^{2}$ Amer Durrani, ${ }^{2}$ Pippa Corrie ${ }^{3}$
}

${ }^{1}$ University of Cambridge School of Clinical Medicine, Cambridge, UK

${ }^{2}$ Department of Plastic and Reconstructive Surgery, Addenbrooke's Hospital, Cambridge, UK ${ }^{3}$ Department of Oncology, Addenbrooke's Hospital, Cambridge, UK

Correspondence to Garance Biosse-Duplan; garance.biosseduplan@ addenbrookes.nhs.uk

Accepted 19 October 2021

Check for updates

(c) BMJ Publishing Group Limited 2021. Re-use permitted under CC BY-NC. No commercial re-use. See rights and permissions. Published by BMJ.

To cite: Biosse-Duplan $\mathrm{G}$, Murphy S, Durrani A, et al. BMJ Case Rep

2021:14:e246070

doi:10.1136/bcr-2021-

246070

\section{SUMMARY}

A 57-year-old woman diagnosed with primary melanoma was managed with a wide variety of treatments over 18 years. Given her long history of disease, the array of therapies she has received range from those no longer recommended to those recently approved. This case highlights the extraordinary rate at which both the medical and surgical melanoma treatment landscape has evolved, alongside how professional consensus has changed over the past two decades. It also demonstrates the innovation and collaboration required between the patient and the multidisciplinary team, as well as how external factors such as national guidelines, eligibility for clinical trials and drug funding in the National Health Service (NHS) alter a management plan, presenting yet another set of challenges when managing cancer patients in the modern era.

\section{BACKGROUND}

Melanoma is a public health concern, with incidence increasing faster than any other type of cancer, affecting both men and women of all ages. ${ }^{1}$ Melanoma is the most lethal form of skin cancer however, the overall 5 -year survival rate has increased dramatically from $52 \%$ to $90 \%$ since the 1970s, while 10-year survival has also almost doubled over the same time period. ${ }^{2}$ The vast majority of people diagnosed with melanoma present with a pigmented lesion and much of the survival gains have been due to earlier diagnosis and intervention with surgical resection. Improving outcomes for patients with advanced melanoma has proved far more challenging, with life expectancy remaining under 1 year for most people well into the 21 st century.

The melanoma treatment landscape has undergone constant and dynamic evolution over the last 20 years, ${ }^{3}$ as historic approaches were learnt from and replaced, while new knowledge and interventions have emerged. Translation of basic and clinical research into changing practice requires significant collaboration from multiple stakeholders involved in healthcare, including pharma, regulators, commissioners, doctors and not least the patients themselves. The most noteworthy gain has been the rapid introduction of several new systemic therapies shown for the first time to improve survival of metastatic melanoma patients into routine clinical practice, which has tripled life expectancy in the last decade, now offering potential for cure in some cases.

We report a patient originally diagnosed with primary melanoma 18 years ago, whose treatment history is a reflection of how rapidly the treatment landscape has changed in both surgery and drug therapy. The case further demonstrates the innovation and collaboration between the patient and healthcare professionals needed to manage multiple challenges that arise along the patient journey.

\section{CASE PRESENTATION}

A 57-year-old Caucasian woman was originally diagnosed with an acral lentiginous melanoma on the left heel (Breslow thickness $5.2 \mathrm{~mm}$ ) while living in Spain in December 2003, which was removed surgically. The initial surgery was followed by a wide local excision and an immediate elective left inguinal lymph node dissection. She received standard high-dose adjuvant interferon over the following year. ${ }^{4}$

In January 2007, multiple subcutaneous intransit metastases occurred, which were resected from her left leg. However, they reoccurred and became too numerous for further surgical clearance, so she was treated with combination chemotherapy comprising cisplatin, vinblastine and dacarbazine. The disease responded well to chemotherapy and was kept under control for several years.

In July 2011, a routine surveillance CT scan identified liver and lung metastases. She returned to the UK, where her tumour was tested for the presence of and confirmed to harbour a BRAFV600 gene mutation. She enrolled in a clinical trial testing a novel combined BRAF-MEK inhibitor drug. The disease regressed on treatment, which was continued for 12 months despite some unpleasant but non-life-threatening side effects. However, in 2012, a restaging scan confirmed enlarging liver metastases, so the trial drug was halted. At this time, a new immune checkpoint inhibitor antibody targeting the CTLA-4 T cell receptor, called ipilimumab, had just been approved for use by the National Institute for Health and Care Excellence (NICE) following international randomised clinical trials which reported overall survival benefit from systemic therapy for the first time ever in metastatic melanoma patients. ${ }^{5}$ The patient was treated with ipilimumab, given over a 12 -week period. Despite risk of complex immune-related side effects, she tolerated it well and she achieved a durable response, lasting 2 years (figure 1 ).

During 2014, the patient developed multiple small soft tissue metastases, which were visible and palpable in various parts of her body, including limbs, groins, axillae and posterior chest wall. As there was no new visceral disease at this time, the larger tumours were managed by surgical resection. 

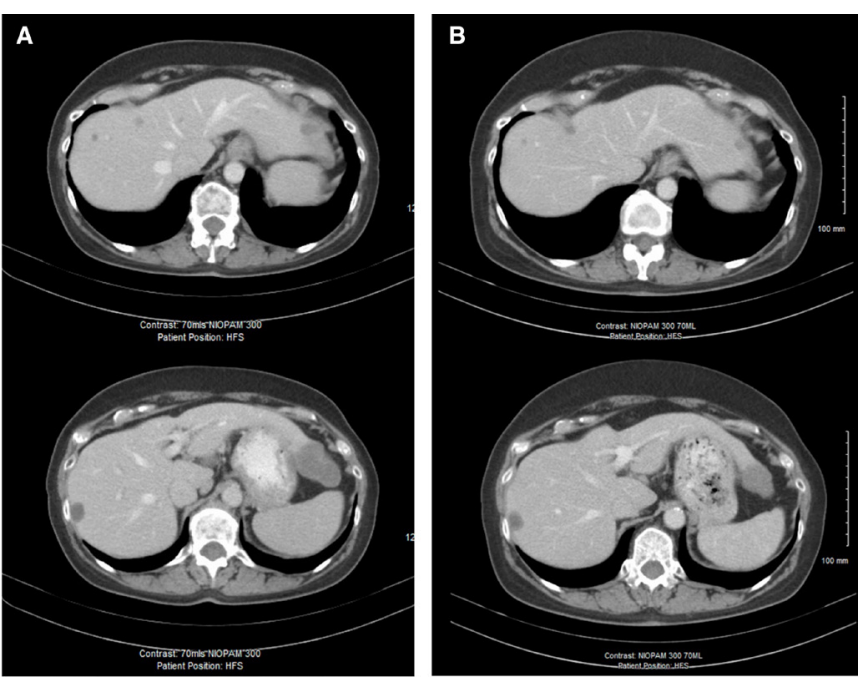

Figure 1 CT scans show low volume liver metastases prior to starting ipilimumab (A) and after 2 years on the drug (B). They remained unchanged.

In 2015, NICE approved a second immune checkpoint inhibitor, pembrolizumab, which blocks the $\mathrm{T}$ cell $\mathrm{PD}-1$ receptor and had been shown to have both superior efficacy and better side effect profile compared with ipilimumab. ${ }^{6}$ A CT scan in December 2015 showed disease progression involving major organs, so the patient began treatment with pembrolizumab in January 2016. The liver and lung metastases were stabilised and she continued on immunotherapy for the next 18 months. During this time, further soft tissue metastases occurred, which were either surgically excised, or palliated with external beam radiotherapy. In 2017, she also underwent electrochemotherapy to manage particularly problematic large volume, painful chest wall soft tissue disease (figure 2) which had not responded to radiotherapy, to good effect and palliation of symptoms.

Unfortunately, in July 2017, the patient developed immunotherapy-related colitis and the pembrolizumab was halted. Once recovered, because it was known that her melanoma harboured a BRAFV600 mutation, she was started on the BRAF inhibitor, dabrafenib, which had been approved by NICE in 2014 . $^{7}$ Her remaining disease responded well, with partial resolution of some soft tissue and visceral metastases (figure 3). New randomised trials reported that combining the MEK inhibitor, trametinib, with dabrafenib, offered greater survival benefit compared with dabrafenib alone, so when NICE approved the combination regimen 5 months later in early 2018, trametinib

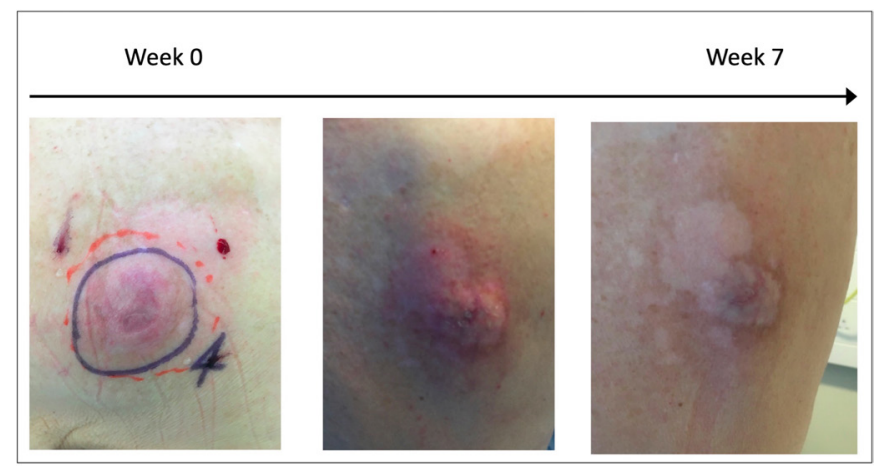

Figure 2 Electrochemotherapy to a posterior chest wall melanoma metastasis generated tumour regression 7 weeks after treatment.
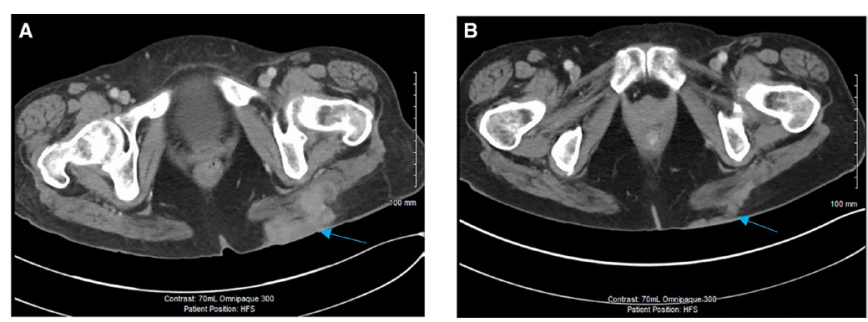

Figure 3 CT scans show a partial response of left buttock soft tissue metastasis (labelled) to dabrafenib before starting $(A)$ and 8 weeks after being on dabrafenib treatment (B).

was added to her treatment regimen. ${ }^{8}$ She went on to achieve an almost complete response on this combination regimen. However, drug treatment was interrupted 6 months later due to intolerable side effects, including uveitis, arthralgia and severe fatigue. In the absence of BRAF inhibition, more subcutaneous metastases recurred, so she was restarted on a modified dose of dabrafenib monotherapy taken intermittently, which she was able to tolerate and kept her disease under control. ${ }^{9}$

A timeline summarising the patient's treatment alongside key practice-changing events in melanoma management is provided in figure 4.

\section{OUTCOME AND FOLLOW UP}

In February 2020, while still continuing in response on intermittent dabrafenib dosing, the patient suffered a debilitating ischaemic stroke, which was unrelated to melanoma. After a period of partial recovery, she restarted dabrafenib, which enabled her to live alongside her disease and maintain a good quality of life despite gradual loss of disease control, with multiple metastases evident on imaging in February 2021. She stopped treatment completely in March 2021 and was admitted for end-of-life hospice care in July 2021.

\section{DISCUSSION}

This case demonstrates the rapid pace at which the melanoma treatment landscape has advanced, particularly during the last decade. With 5-year and 10-year survival rates increasing, metastatic melanoma is becoming a chronic disease rather than a death sentence, so awareness of the increasing treatment options at our disposal is all the more important.

When our patient was first diagnosed, surgery was the only treatment for melanoma known to improve survival. Adjuvant interferon was approved for use in the USA, but with uncertain benefits alongside significant toxicity. ${ }^{10} 11$ Dacarbazine was used to treat metastatic disease, offering a modest response rate only and median overall survival of patients diagnosed with advanced melanoma was around 8 months. In the early 21 st century, huge progress in our understanding of cancer at the molecular level led to a revolution of mechanism-driven anticancer therapies being developed. This approach has been particularly successful in melanoma, now with two major new forms of systemic therapy established as standard of care: immune checkpoint inhibitor antibodies (eg, pembrolizumab, nivolumab and ipilimumab), as well as small molecule inhibitors of the BRAF-MEK signalling pathway (eg, vemurafenib, dabrafenib and trametinib) for those $50 \%$ of patients with BRAF mutant melanoma. Modern systemic therapy has extended median overall survival of metastatic melanoma to beyond 3 years, with many patients entering long term remission, some of whom may yet be considered cured. 


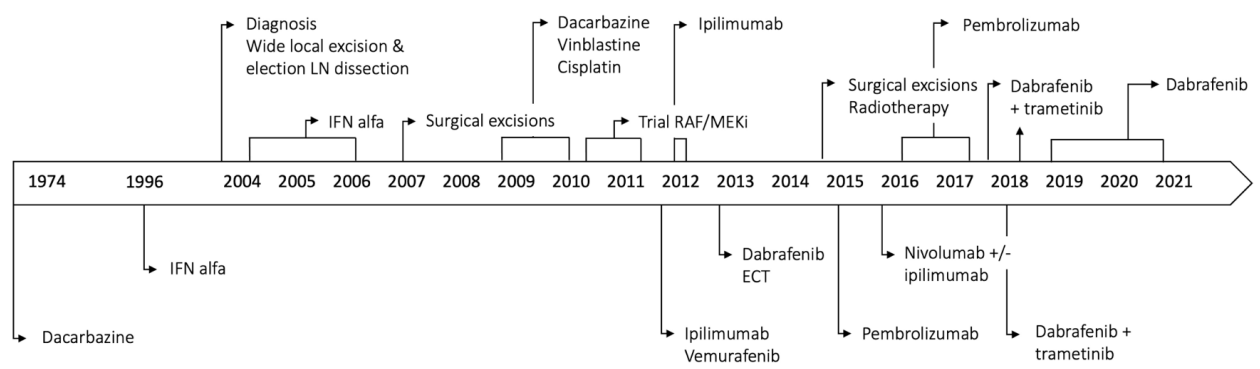

Figure 4 Timeline showing key interventions during the patient pathway (above the timeline) and the availability of standards of care associated with NICE guidelines (below the timeline). Note, access to dacarbazine and IFN alpha predate NICE. IFN, interferon; LN, lymph node; RAF/MEKi, RAF/ MEK protein kinase inhibitor; ECT, electrochemotherapy

With new treatments come new challenges, including how best to sequence and combine different classes of anticancer drugs, as well as how to manage complex toxicities entirely different to those associated with traditional cytotoxic chemotherapy. Of note, while BRAF-targeted therapies tend to generate relatively minor but chronic side effects, immune-related toxicities can be both life-threatening and life-changing. Immune-mediated inflammation may require hospitalisation and support by intensivists across multiple specialties, while some data are emerging which links severe toxicity to a greater chance of treatment response. ${ }^{12} 13$ There is a pressing need to educate non-oncologists regarding this new class of anticancer drugs which are being used to treat increasing numbers of cancer types. Importantly, we also have yet to assess the impact on these new agents in long-term survivors.

In recent years, both immune checkpoint inhibitors and BRAF targeted therapy have been tested as adjuvant therapy and have been shown to halve the rate of recurrence when given to patients with high risk resected melanoma for up to 1 year after their surgery. ${ }^{8} 14-16$ Thus the historical use of adjuvant interferon can finally be put to bed.

Despite the excitement around novel systemic therapies, the mainstay of treatment for most patients diagnosed with melanoma remains primary surgical resection. Management of regional lymph nodes has evolved over the last decade. As was standard practice in the late 20th century, our patient had an immediate inguinal lymph node dissection at the time of primary melanoma resection, aimed at preventing disease spread. Regional lymph node clearance is associated with significant morbidity, including potentially debilitating lymphoedema of the affected limb. A randomised trial failed to show any survival gain with this approach, ${ }^{17}$ which led to the practice being abandoned in the 21st century. On the other hand, techniques to identify the sentinel lymph node were developed and sentinel lymph node biopsy (SLNB) has been adopted into routine clinical practice. ${ }^{18}$ SLNB provides a key pathological staging tool, but the question whether removal of the sentinel node offered therapeutic benefit has been the subject of great debate. SLNB positive patients were previously offered completion lymph node dissection (CLND), but three landmark trials have now convincingly shown no melanoma-specific survival benefit of full lymph node clearance. ${ }^{19-21}$ Therefore, this practice has now stopped. Instead, modern melanoma surgeons play an increasing role supporting management of metastatic melanoma patients. CLND is indicated for patients presenting with palpable regional lymph nodes. Furthermore, as demonstrated by our patient, differential response to systemic therapy is often seen, with some metastases becoming resistant while others are kept in check. Surgeons assist in removing isolated drug-resistant lesions alongside systemic therapy maintenance. Electrochemotherapy, which our patient received in 2017, was approved by NICE in $2013^{22}$ as an additional innovative intervention available for controlling limited metastatic melanoma where surgery is not practical.

Our case demonstrates how an individual patient diagnosed with melanoma was able to gain benefit from a series of new interventions that became available during the course of her 18-year illness. Alongside her treatment, she was able to maintain a good quality of life, her own expressed wish was to become a grandmother and she achieved and enjoyed this to the full. Her disease was managed through a multidisciplinary team (MDT) of specialists working to good effect. The MDT was able to take advantage of effective international research and clinical trials, as well as guidance from national regulators and commissioners who took bold decisions at the time to provide patients with significant unmet need access to high cost drugs, based on sound health economic assessments confirming their cost-effectiveness.

Patient's perspective

During my long and varied 'assault' on my disease, I always felt that every day of my survival, was 1 day closer to the eventual discovery through constant research of a cure. I felt many times that I was living with a chronic disease when receiving a new drug or therapy, and enjoyed a useful and good quality of life for much of the time. A minor but important point to my own wellbeing is that I have never worried about what tomorrow may or may not bring, and only put my energies into what challenges today presents.

\section{Learning points}

- The melanoma treatment landscape of melanoma has been radically transformed this century and particularly over the last decade.

- The impact of both new non-surgical therapies and surgical interventions has extended life expectancy with improved quality of life at all stages of disease.

- Long-term benefits of adjuvant systemic therapy suggest recurrence rates can be halved, while some metastatic melanoma patients are likely being cured.

- Optimal care requires a well-informed, multidisciplinary team to co-ordinate individual patient management.

Acknowledgements The authors would like to thank Ms Victoria McMorran for her invaluable role in the patient's care. 
Contributors Conceptualised and supervised by AD. The patient was under care of PC, AD and SM. Report was written by GB-D, with invaluable help from PC and SM.

Funding The authors have not declared a specific grant for this research from any funding agency in the public, commercial or not-for-profit sectors.

Competing interests None declared.

Patient consent for publication Consent obtained directly from patient(s) Provenance and peer review Not commissioned; externally peer reviewed.

Open access This is an open access article distributed in accordance with the Creative Commons Attribution Non Commercial (CC BY-NC 4.0) license, which permits others to distribute, remix, adapt, build upon this work non-commercially, and license their derivative works on different terms, provided the original work is properly cited and the use is non-commercial. See: http://creativecommons.org/ licenses/by-nc/4.0/.

\section{ORCID iD}

Garance Biosse-Duplan http://orcid.org/0000-0002-6685-8574

\section{REFERENCES}

1 Common cancer sites - cancer STAT facts. Available: https://seer.cancer.gov/statfacts/ $\mathrm{html} /$ common.html [Accessed $20 \mathrm{Jul}$ 2021].

2 Melanoma skin cancer survival statistics [Internet]. Cancer Research UK, 2015. Available: https://www.cancerresearchuk.org/health-professional/cancer-statistics/ statistics-by-cancer-type/melanoma-skin-cancer/survival [Accessed 15 Jan 2021].

3 Curti BD, Faries MB. Recent advances in the treatment of melanoma. Longo dl, editor. N Engl J Med 2021;384:2229-40.

4 Kirkwood JM, Manola J, Ibrahim J, et al. A pooled analysis of eastern cooperative Oncology group and intergroup trials of adjuvant high-dose interferon for melanoma. Clin Cancer Res 2004:10:1670-7.

5 National Institute for Health and Care Excellence. Ipilimumab for previously treated advanced unresectable or metastatic melanoma (Technology appraisal guidance [TA268]), 2012. Available: https://www.nice.org.uk/guidance/ta268

6 National Institute for Health and Care Excellence. Pembrolizumab for treating advanced melanoma after disease progression with ipilimumab (Technology appraisal guidance [TA357]), 2015. Available: https://www.nice.org.uk/guidance/ta357

7 National Institute for Health and Care Excellence. Dabrafenib for treating unresectable or metastatic BRAF V600 mutation-positive melanoma (Technology appraisal guidance [TA321]), 2014. Available: https://www.nice.org.uk/guidance/ta321

8 National Institute for Health and Care Excellence. Dabrafenib with trametinib for adjuvant treatment of resected BRAF V600 mutation-positive melanoma (Technology appraisal guidance [TA544]), 2018. Available: https://www.nice.org.uk/guidance/ ta544

9 Dooley AJ, Gupta A, Bhattacharyya M, et al. Intermittent dosing with vemurafenib in BRAF V600E-mutant melanoma: review of a case series. Ther Adv Med Oncol 2014;6:262-6.

10 Cole BF, Gelber RD, Kirkwood JM, et al. Quality-of-life-adjusted survival analysis of interferon alfa-2b adjuvant treatment of high-risk resected cutaneous melanoma: an eastern cooperative Oncology Group study. J Clin Oncol 1996;14:2666-73.

11 Tarhini AA. The current state of adjuvant therapy of melanoma. Lancet Oncol 2020:21:1394-5.

12 Das S, Johnson DB. Immune-Related adverse events and anti-tumor efficacy of immune checkpoint inhibitors. J Immunother Cancer 2019;7:306.

13 Dimitriou F, Staeger R, Ak M, et al. Frequency, treatment and outcome of immunerelated toxicities in patients with Immune-Checkpoint inhibitors for advanced melanoma: results from an institutional database analysis. Cancers 2021;13:2931.

14 National Institute for Health and Care Excellence. Pembrolizumab for advanced melanoma not previously treated with ipilimumab (Technology appraisal guidance [TA366]), 2015. Available: https://www.nice.org.uk/guidance/ta366

15 National Institute for Health and Care Excellence. Nivolumab for treating advanced (unresectable or metastatic) melanoma (Technology appraisal guidance [TA384]), 2016. Available: https://www.nice.org.uk/guidance/ta384

16 National Institute for Health and Care Excellence. Systemic anticancer therapies for stage IV melanoma (NICE pathways), 2021. Available: https://pathways.nice.org.uk/ pathways/melanoma

17 Cascinelli N, Morabito A, Santinami M, et al. Immediate or delayed dissection of regional nodes in patients with melanoma of the trunk: a randomised trial. The Lancet 1998;351:793-6.

18 Morton DL, Thompson JF, Essner R, et al. Validation of the accuracy of intraoperative lymphatic mapping and sentinel lymphadenectomy for early-stage melanoma. Ann Surg 1999;230:453.

19 Morton DL, Elashoff R, Hoekstra HJ. Sentinel-Node biopsy or nodal observation in melanoma. $n$ engl $j$ med 2006;11.

20 Leiter U, Stadler R, Mauch C, et al. Complete lymph node dissection versus no dissection in patients with sentinel lymph node biopsy positive melanoma (DeCOG-SLT): a multicentre, randomised, phase 3 trial. Lancet Oncol 2016;17:757-67.

21 Faries MB, Thompson JF, Cochran AJ, et al. Completion dissection or observation for sentinel-node metastasis in melanoma. N Eng/ J Med 2017;376:2211-22.

22 National Institute for Health and Care Excellence. Electrochemotherapy for metastases in the skin from tumours of non-skin origin and melanoma (Interventional procedures guidance [IPG446]), 2013. Available: https://www.nice.org.uk/quidance/ipg446

Copyright 2021 BMJ Publishing Group. All rights reserved. For permission to reuse any of this content visit https://www.bmj.com/company/products-services/rights-and-licensing/permissions/

BMJ Case Report Fellows may re-use this article for personal use and teaching without any further permission.

Become a Fellow of BMJ Case Reports today and you can:

- Submit as many cases as you like

- Enjoy fast sympathetic peer review and rapid publication of accepted articles

Access all the published articles

Re-use any of the published material for personal use and teaching without further permission

Customer Service

If you have any further queries about your subscription, please contact our customer services team on +44 (0) 2071111105 or via email at support@bmj.com.

Visit casereports.bmj.com for more articles like this and to become a Fellow 